\title{
Quality standards and samples in genetic testing
}

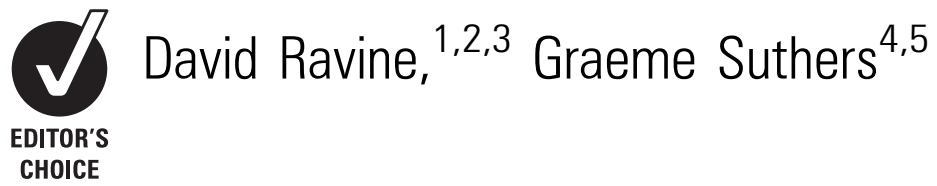

The most critical performance indicator for medical laboratories is the delivery of accurate test results. In any laboratory, there is always the possibility that random or systematic errors may occur and place human health and welfare at risk. Laboratory quality assurance programmes continue to drive improvements in analytical accuracy. The most rigorously scrutinised data on laboratory errors, which come from transfusion medicine, reveal that the incidence of analytical errors has fallen to levels where most of the residual risk is now found in preanalytical links in the chain from patient to result, particularly activities associated with ordering of tests and sample collection. This insight is important for genetic testing because, like pretransfusion testing of patients with unknown blood groups, a substantial proportion of genotyping results cannot be immediately verified. An increasing number of clinical decisions, associated personal and social choices, and legal outcomes are now influenced by genetic test results in the absence of other confirmatory data. An incorrect test result may lead to unnecessary and irreversible interventions, which may in themselves have associated risks for the patient, inaccurate risk assessment regarding the disease, missed opportunities for disease prevention or even wrongful conviction in a court of law. Unfortunately, there is limited information available about the risk of preanalytical errors associated with, and few published guidelines regarding, sample collection for genetic testing. The growing number and range of important decisions made on the basis of genetic findings warrant a reappraisal of current standards to minimise risks in genetic testing.

Errors are an established, unwelcome feature in all areas of healthcare. ${ }^{1}$ Erroneous test results are widespread ${ }^{2}$ and are a recognised cause of iatrogenic harm. Incorrect genetic test results take the issue further. Besides introducing risks of unnecessary and irreversible interventions for those being tested, they may also lead to missed opportunities for disease prevention among relatives. With genetics emerging as a substantial discipline within pathology and laboratory medicine, there is a responsibility now to reassess current standards for minimising risks of error in the field.

As with all pathology investigations, errors may occur at any point in the

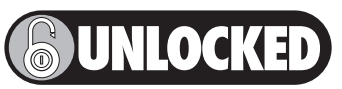

This paper is freely available online under the BMJ Journals unlocked scheme, see http://jcp.bmj.com/site/ about/unlocked.xhtml

${ }^{1}$ School of Pathology and Laboratory Medicine; ${ }^{2}$ Western Australian Institute of Medical Research, University of Western Australia, Perth, Western Australia, Australia; ${ }^{3}$ PathWest, OEIl Medical Centre, Nedlands, Western Australia, Australia; ${ }^{4}$ Department of Paediatrics, University of Adelaide, Adelaide, South Australia, Australia; ${ }^{5}$ Royal College of Pathologists of Australasia, Sydney, New South Wales, Australia

Correspondence to Dr Graeme Suthers, SA Clinical Genetics Service, SA Pathology, Women's \& Children's Hospital, North Adelaide, SA 5006, Australia;

graeme.suthers@health.sa.gov.au genetic test processing chain from test selection by the clinician to clinical decisions based on the result. ${ }^{3}$ Although the iterative improvements in proficiency testing by molecular genetic testing laboratories have been encouraging, ${ }^{4}$ they are also a reminder that genetic testing is not immune from the usual risks of error.

\section{RISKS ASSOCIATED WITH GENETIC TESTING}

Testing for medically significant genetic variants is growing rapidly, although it is still a small component of medical testing overall. In the UK in 2005-2006, genetic tests accounted for $<0.1 \%$ of all laboratory investigations. ${ }^{6}$ Compared with their working familiarity with most pathology investigations, many clinicians still have little experience of testing for inherited or de novo genomic variants.

As well as the usual challenges associated with bringing new technologies into healthcare, there are further issues associated with genetic investigations to consider. Genetic findings may aid the task of assessing clinical symptoms. They may also confirm a diagnosis long before the onset of symptoms in an individual patient. This is especially the case for inherited disorders with readily identifiable biomarkers. For example, serum cholesterol testing of relatives at risk of familial hypercholesterolaemia has long been known to refine or resolve genetic risks identified by family history. The advent of genetic testing for this disorder in families for whom the causal mutation has been identified has led to improved diagnostic accuracy and simplified the process of risk assessment among relatives.?

Genetic testing is increasingly requested for healthy people identified by family history as being at high risk of having inherited a mutation predisposing to a serious adult-onset autosomal dominant monogenic disorder. Many of these conditions have no associated subclinical manifestations. Referred to as predictive, presymptomatic or predisposition testing, this category of testing is distinguished by absence of corroborating clinical or other confirmatory data. This scenario is not unique to late-onset dominant disorders and is often seen in other areas of genetic testing including prenatal, preimplantation genetic diagnosis, and carrier testing for autosomal and $\mathrm{X}$ linked recessive disorders. It is also a prominent feature of 'personalised medicine', ${ }^{8}$ where genotyping is used to predict responses to, or side effects from, drugs.

Corroboration of evidence is an important aspect of clinical care. For example, a normal chest radiograph from a patient with severe congestive cardiac failure would normally prompt a check to ensure the correct image is being viewed. Many clinical measurements are corroborated by repeat measurements. The measurement of blood pressure, for example, can identify patients at increased risk of stroke. Although no other clinical features may be available to corroborate the result, repeated measurements over time provide a background in which outlying or incongruent findings can be evaluated.

Now, consider the case of testing a DNA sample from a young woman for a BRCA1 mutation, which was previously identified in her mother, who has a strong family history of early onset breast cancer. The investigation will yield a result that is qualitatively different from the outputs of most clinical investigations. The woman's family history alone will have identified her as having a 1 in 2 risk of inheriting the mutation and, in contrast to many medical test results, the outcome is binary. The mutation is either present, in which case she has a high lifetime risk of breast and ovarian cancer, or absent, with her cancer risk returning to the background population level for women of her age. Unless verified by replicate testing, there are usually no further opportunities to 
Case study

A 40-year-old male patient was diagnosed with an extra-adrenal paraganglioma. Family history revealed a fourth degree relative who presented with medullary thyroid cancer at 25 years of age. Early-onset medullary thyroid cancer is the hallmark of multiple endocrine neoplasia type 2, which is an autosomal dominant disorder due to mutations in the RET gene. Some mutation carriers remain healthy, but their risk of developing thyroid cancer is high. RET mutations can also be associated with paragangliomas. The patient was tested for a heritable RET mutation by an accredited laboratory, and a well-recognised pathogenic mutation was identified. He proceeded to have a prophylactic thyroidectomy, which is the recommended management for mutation carriers. The patient's unaffected children subsequently had predictive genetic testing and none was identified with the mutation. The relative with thyroid cancer was eventually reviewed and testing demonstrated that she did not have our patient's RET mutation. Instead, a different mutation was identified in the same gene. Repeat sample collection and analysis demonstrated that the RET mutation originally identified in the index patient was a laboratory artefact. The revised result eliminated the earlier justification for his prophylactic thyroidectomy and also for the predictive genetic testing of his children. He was subsequently shown to have a heritable mutation in the SDHD gene. Although the amended diagnosis also has familial implications, his children are now disenchanted and have declined further involvement with the clinic.

corroborate the finding. Furthermore, the test result and any associated clinical or psychosocial sequelae are very likely to remain unchallenged over a lifetime, and possibly beyond into the next generation. In this setting, the risk of error in genetic testing is not merely academic. Many genetic testing services have their own anecdotes and, with permission from the patient involved, we include an example from our own experience (see case study).

The case demonstrates that genetic testing errors can have wide ranging and lasting sequelae. For example, the fact our patient did not develop thyroid cancer could well have been erroneously attributed in the future to the 'success' of his thyroidectomy, when the real reason was his risk was negligible in the first place. Although the consequences of a genetic testing error can have no discernible effect, particularly if the error remains undetected, a range of outcomes is possible, including occasional severe harm or avoidable death.

\section{SOURCES AND FREQUENCY OF GENETIC TESTING ERRORS}

The error we reported was shown to have occurred during the analytical phase of testing. However, it could just as easily have occurred before the sample arrived in the laboratory or postanalysis. Analytical errors have been the prime focus of molecular genetic quality assurance ( $O A$ ) programmes developed by professional bodies in the USA, Europe and Australasia. ${ }^{9-13}$ While the success of these programmes is well documented, most preanalytical risks, particularly those associated with the ordering of tests and sample collection, are beyond their scope.

There are very few published reports about the incidence of errors in genetic testing. One report documented 1.7 errors per 1000 investigations (excluding errors in test selection and interpretation). ${ }^{14}$ Errors were equally divided between preanalytical (sample collection and handling) and analytical phases. A useful insight from this audit is that the reported error rate is in the same range as those reported in other types of medical testing. ${ }^{15}$ In fact, the occult nature of an erroneous predictive genetic test result, as illustrated in the case study, demonstrates that estimates of the prevalence of adverse consequences associated with predictive testing are best derived from independent corroborating data. However, these data can only come from resampling and retesting and, as far as we are aware, they are scanty and anecdotal.

\section{INSIGHTS FROM OTHER AREAS OF LABORATORY MEDICINE}

In contrast to the likelihood that a proportion of genetic testing errors will remain undetected, errors associated with allogenic blood transfusion or tissue transplantation are rapidly unmasked. Life-threatening immune reactions to mismatched red cell and tissue immunotypes have focused attention on the incidence and sources of errors, and also on strengthening risk minimisation strategies.

Studies of pretransfusion testing report that $0.5-0.8$ sample tubes per 1000 contain blood from someone other than the identified patient, often referred to as "wrong blood in the tube' (WBIT). ${ }^{16} 17$ They also reveal other risks, including incomplete labelling in approximately 11 samples per $1000{ }^{16}{ }^{18}$ Such labelling has been shown to result in a 40-fold increased risk of sample errors compared with the risk associated with properly labelled samples. ${ }^{19}$

There are no comparable data for genetic testing. It is important to recognise, however, that current reported pretransfusion error rates are likely to be lower than those prevailing in the era before there was heightened emphasis on risk minimisation strategies. ${ }^{20}$ It is possible that the situation with genetic testing may be more hazardous as there are additional known risks associated with sample collection. These include labelling of samples collected from interventional procedures, ${ }^{21}$ such as amniotic fluid, chorionic villus and fetal blood sampling, from patients in delivery rooms ${ }^{22}$ and synchronously from relatives. ${ }^{23}$

Analytical error rates in pretransfusion testing have now fallen to levels where most residual mistakes occur in preanalytical phases, particularly those associated with blood sample collection and labelling. The same trend is well documented in other general laboratory arenas. ${ }^{24} 25$ It is notable that a range of error-prone preanalytical tasks are often completed by people beyond the jurisdiction of laboratories, including referring clinicians, clients and carers as well as workers contributing to data entry, specimen collection and transport.

\section{RISKS OF PREANALYTICAL ERRORS IN GENETIC TESTING}

Acknowledgement of risks of preanalytical errors, particularly sample mislabelling, raises the question whether the safety of patients undergoing genetic testing is adequately protected by the current scope of genetic testing OA programmes.

The prospect of a mislabelled sample destined for genetic testing highlights an additional important risk associated with an adverse outcome. Medical testing is generally prompted by a patient's history or symptoms indicating an increased prior probability of an underlying disease process. In the case of predictive genetic testing, the investigation is prompted by a family history indicating a high risk of predisposition to future illness from an inherited gene mutation. The prior risk of abnormality in a mislabelled sample defines the likelihood that a result will differ from the patient's true status. For example, if 1 in 20 samples from a patient cohort have abnormal test results, then analyses of swapped samples will yield correct results 
for both patients more than $90 \%$ of the time. Alternatively, an inadvertent switch of samples from individuals in whom the prior risk of abnormality is 1 in 2 , which is the case among siblings at risk of having inherited a mutation from an affected parent, will yield correct results for both individuals only $50 \%$ of the time.

In the context of a family history of disease, the elevated prior probability of having inherited the disease-causing mutation also raises the risk of harm arising from sampling errors. This risk is compounded by the earlier mentioned evidence of increased risk of sample mislabelling associated with synchronous sample collection from relatives. ${ }^{23}$

Although some indication of the likely background error rate associated with predictive genetic testing would be useful, any estimate will inevitably be the average of numerous random and systematic error rates, all of which will also be inevitably subject to regional variation and changes over time. Notwithstanding these limitations, it is apparent that the current ad hoc approach to minimising preanalytical risks associated with genetic testing, particularly the risk of sampling errors, is a patient safety issue that requires research attention. Meanwhile, it is important that those involved in this area of medicine consider all potential weaknesses in the total testing process, and then avail themselves of the full range of available risk minimisation strategies.

\section{GRADIENT OF RISK MANAGEMENT STRATEGIES}

Experience from transfusion and transplantation medicine provides guidance on risk minimisation strategies for genetic testing. These services have implemented measures over many years to decrease the incidence of sampling errors. Importantly, both these services reject the baseline standard of analysing single specimens that fulfil minimum labelling criteria, such as concordance of two person-specific identifiers on the sample tube and accompanying request form. ${ }^{26}$

To ensure against transfusing the wrong blood into a patient, transfusion services use a range of strategies which, in general terms, have focused on strengthening the chain of custody procedures in lieu of analysis of a second independently drawn sample. Measures include requiring a second person to confirm concordance of patient identity, request form and sample; avoiding prelabelled tubes; appointing safety officers to oversee training and processes; and using electronic patient identification systems. A multicentre international survey of 650000 samples reported in 2003 that 6.1 samples per 1000 were mislabelled $^{16}$ and a WBIT rate of 0.5 samples per 1000, despite almost all participating laboratories having written policies on sample collection and labelling. ${ }^{27}$ The Division of Transfusion Medicine at the University of California, Los Angeles, as a result of a 17-year policy of requiring two independent samples for blood typing before issuing type-specific blood for non-group- $\mathrm{O}$ patients, reported a very similar mislabelling rate of 0.44 samples per $1000 .^{22}$ In keeping with the policy of the University of California, Los Angeles, the World Marrow Donor Association also established guidelines recommending confirmatory tissue typing on duplicate samples from potential donors and recipients before transplantation. ${ }^{28}$ The policy of confirmatory testing of a second independent blood sample for patients without a historical record corroborating an $\mathrm{ABO}$ typing result from a single sample has since been adopted by more transfusion services and now extends to approximately $20 \%$ of US laboratory and hospital practices. ${ }^{18}$

\section{CURRENT SAMPLE VERIFICATION PROTOCOLS IN GENETIC TESTING}

Although there has been a long-standing focus on reducing the risk of sampling errors associated with transfusion and transplantation, most contemporary reports $^{29} 30$ and documents on genetic testing developed by regulatory and professional bodies ${ }^{31-38}$ have primarily addressed strengthening laboratory OA. With some notable exceptions, ${ }^{39} 40$ few have argued in favour of adopting a more patient-centric approach to risk minimisation, including addressing risks associated with sample collection. A survey of directors of 17 medical genetic laboratories in Australia and New Zealand in 2005, ${ }^{41}$ before sampling risk minimisation guidelines were implemented, revealed a range of approaches. In lieu of duplicate sampling, some laboratories split samples for predictive testing into two tubes immediately on arrival, and then arranged independent replicate analyses. However, most laboratories accepted single unsigned samples for predictive genetic testing after ensuring concordance of patient identifying details on request forms and samples. Subsequently, an Australian laboratory accreditation guideline, which is not obligatory, was issued in 2006 recommending that predictive genetic tests be conducted on duplicate samples. ${ }^{42}$

\section{VERIFYING GENETIC TEST RESULTS}

Genetic testing is increasingly used to resolve clinical uncertainty in a diverse array of medical scenarios. Many scenarios offer opportunities to corroborate genetic test findings. For example, a test report that a child has mutations in both CFTR genes may be verified by a pre-existing clinical diagnosis of cystic fibrosis (an autosomal recessive disorder caused by CFTR mutations), by finding an elevated sweat chloride concentration (a specific biochemical feature of cystic fibrosis) or by demonstrating that the parents are carriers of the child's mutations. As another example, an array genomic scanning result in a sample from a 3 -year-old child with autism might unexpectedly demonstrate a genomic deletion involving the TP53 gene. The finding may prompt a discovery of a previously unrecognised family history indicative of $\mathrm{Li}$ Fraumini syndrome. However, as the case study we present illustrates, the family history may be misleading, inconclusive or absent, in which case duplicate sampling would avert the risk of arranging inappropriate predictive genetic tests for relatives. The number of confirmatory options already available demonstrate the usefulness of an informed and practical approach to risk minimisation in genetic testing, particularly in the absence of peer reviewed data and $\mathrm{OA}$ programmes that focus on the quality of all links in the chain from patient to result.

\section{RISK MINIMISATION STRATEGIES FOR GENETIC TESTING}

There is at the very least an ethical imperative that any genetic test finding with the potential to influence significant clinical, personal or social decisions should prompt consideration of all associated risks, including the risk of an erroneous result. A recent report to the Victorian Department of Justice ${ }^{43}$ offers an illuminating insight into contemporary societal views on the strength of DNA evidence. An inquiry into the wrongful conviction of a man for rape in July 2008 identified that the conviction had hinged on DNA evidence derived from a single sample. At the time of the man's conviction, it had not been appreciated that the sample had been collected in the same unit as forensic samples secured by the same doctor $30 \mathrm{~h}$ earlier from another woman, with whom, it was undisputed, the man had had prior sexual contact. A former judge who conducted the review warned:

the DNA evidence had been perceived as being so powerful by all involved in the 
case that none of the filters upon which our system of criminal justice depends to minimise the risk of a miscarriage of justice, operated effectively at any stage until a matter of weeks before [the man's] appeal was expected to be heard.

The fact an innocent man spent time in jail as a convicted rapist on the basis of an uncorroborated genetic identity test result is a sobering reminder of the extent of potential 'fall out' when things go wrong in genetic testing. Clearly, such errors are not solely a concern for medical providers. Sensibly, the importance of corroborating a genetic test result will vary as will the best ways of achieving verification. Ideally, consideration should be made of the likely risk of error; the seriousness and frequency of any possible adverse consequences; and the range, effectiveness and total cost of available risk minimisation strategies. Examination of legal and financial risks for those who are professionally accountable may also need to be included in the cost analysis.

There is a strong argument now that healthcare practitioners involved in requesting or performing genetic tests which have potentially fatal outcomes associated with sampling errors should recognise that the baseline standard of analysing single specimens fulfilling minimum labelling criteria represents inadequate risk minimisation for the patient. ${ }^{44} 45$ The full scope of potentially fatal outcomes must also be considered, including any risk of future fatalities linked to missed opportunities for disease prevention among relatives. Further, given the range of deleterious non-fatal outcomes associated with genetic testing, there is a case for extending this recommendation to incorporate significant clinical, personal, social or legal decisions that may be directed by a genotype finding.

There are, however, several additional measures to consider in relation to genetic testing. For example, the option of routine replicate testing on independent samples has long been regarded as inappropriate for prenatal genetic diagnosis, particularly if the invasive procedure required to obtain a second sample has attendant potentially serious risks, either for the mother or for the fetus. Fortunately, in this arena, simultaneous testing of nondisease associated genetic polymorphisms in fetal and parental DNA samples is an increasingly available option to detect sample switching, and also to assess for possible maternal cell contamination. ${ }^{46}$

It is worth noting that in other arenas, such as predictive genetic testing for serious late-onset dominant disorders, including familial cancers, late-onset neurodegenerative disorders and hereditary arrhythmia syndromes, some professional organisations ${ }^{47} 48$ have issued guidelines recommending replicate testing. When considering the option of replicate testing, some practitioners may contemplate arranging the test in a second laboratory to provide additional protection against unrecognised laboratory-specific risks of systematic error. This opportunity, however, would not be available for genetic tests that are currently monopolised through patents or licensing.

With ongoing genetic advances, the issue of risk minimisation in relation to genetic testing is now extending into mainstream medical practice. Pharmacogenomic testing has a growing role in guiding therapeutic decisions, including management of hepatitis $C$ virus infection, which is the most common bloodborne infection in the USA. ${ }^{49}$ Until now, there has been little commentary about whether there is a need to strengthen the quality of preanalytical and postanalytical phases associated with pharmacogenomic testing, particularly in the boundary areas linking clinical and laboratory medicine.

While risk minimisation strategies should be tailored to individual clinical scenarios, cost-effectiveness is an important consideration. For example, if we accept that a single sample is appropriate for prenatal testing of fetal tissue, there are few published recommendations regarding the minimum labelling and chain of custody requirements other than in relation to paternity testing. What impact would strengthened risk minimisation measures have on the cost of delivering prenatal testing? And would the extra cost be acceptable to those paying for the service?

In the absence of widely accepted professional guidelines, OA programmes or peer reviewed data for genetic test sampling, the decision to implement a more stringent sample protocol is ultimately directed locally based on what is known about the clinical risks involved. Venesectionists, laboratory scientists and pathologists are not necessarily aware of these details. The ultimate responsibility for individual patient safety is vested in the clinician requesting the investigation. On the other hand, laboratory-based practitioners are well placed to remind referring clinicians of sampling recommendations.

\section{CONCLUSION}

The goal of a clinician is to provide the patient with an accurate diagnosis, prog- nosis and therapeutic options, including in relation to diseases for which genetic tests are available. Similarly, the goal of a medical laboratory is to provide the right result for the right patient in a timely fashion every time. Alexander Pope wrote in An Essay on Criticism that "To err is human...'. Three hundred years later, his message is still potent. All arenas of human endeavour are at risk of human error, and the emerging discipline of genetic testing is not immune. Errors will occur here, as they do in other areas of laboratory testing, and medicine in general. It is of little comfort that sample errors, such as WBIT, are likely to be more common than reports of adverse incidents.

Like the proverbial elephant in the room, we know the errors are present but we hesitate to talk about them. The issue must be addressed, however, because errors in genetic testing have the potential to prompt clinical decisions with a high risk of attendant harm. They may also direct important life choices for those being tested, with ramifications that may influence human health and welfare at all developmental stages. Some errors will invariably lead to outcomes over which the person being tested will have no control, such as wrongful conviction in a court of law. Errors in genetic testing may also waste the increasingly scarce health dollars, and place individual healthcare practitioners at professional, legal and financial risk. It is now time for the profession to consider the full range of errors that are possible along the genetic test processing chain from patient to

Interactive multiple choice questions

This JCP review article has an accompanying set of multiple choice questions (MCQs). To access the questions, click on BMJ Learning: Take this module on BMJ Learning from the content box at the top right and bottom left of the online article. For more information please go to: http:// jcp.bmj.com/education. Please note: The MCOs are hosted on BMJ Learning - the best available learning website for medical professionals from the BMJ Group. If prompted, subscribers must sign into JCP with their journal's username and password. All users must also complete a one-time registration on BMJ Learning and subsequently log in (with a BMJ Learning username and password) on every visit. 
result, and devise appropriate risk minimisation strategies. Until such data are available, individual healthcare practitioners involved in genetic testing should consider the associated possible risks to patient health and welfare, and look beyond the baseline standard of testing a single sample.

Acknowledgements We are grateful to our many colleagues who contributed to a succession of vigorous discussions on this topic. We especially wish to acknowledge those who 'went the extra mile' and provided relevant data, references and opinions, as well as reviewed drafts of the document: Jacqueline Carroll, Ken Davis, Rob Elles, Andrew Fellowes, Jeni Hood, the late Karen Snow-Bailey, Nicole Staples and Michael Watson. Any shortcomings in the presentation of our arguments are our responsibility alone.

Funding The RCPA position statement cited in the article, "Sample Requirements for Medical Genetic Testing", was prepared with support of a grant from the Australian Government's Department of Health \& Ageing The Department had no role in determining the content, conclusions, or distribution of the document. The Department had no role in the preparation of this article.

Competing interests None.

Contributors Both authors conceived and wrote the article.

Provenance and peer review Not commissioned; externally peer reviewed.

Accepted 16 November 2011

Published Online First 18 January 2012

J Clin Pathol 2012;65:389-393.

doi:10.1136/jclinpath-2011-200519

\section{REFERENCES}

1. Brennan TA, Leape LL, Laird NM, et al. Incidence of adverse events and negligence in hospitalized patients: results of the Harvard Medical Practice Study I. N Engl J Med 1991;324:370-6.

2. Howanitz PJ. Errors in laboratory medicine. Arch Pathol Lab Med 2005;129:1252-61.

3. Giardiello FM, Brensinger JD, Petersen GM, et al. The use and interpretation of commercial APC gene testing for familial adenomatous polyposis. $N$ Engl J Med 1997;336:823-7.

4. McGovern MM, Benach MO, Wallenstein S, et al. Quality assurance in molecular genetic testing laboratories. JAMA 1999;281:835-40.

5. Dequeker $\mathbf{E}$, Cassiman JJ. Genetic testing and quality control in diagnostic laboratories. Nat Genet 2000;25:259-60.

6. UK Department of Health. Report of the Review of NHS Pathology Services in England. 2006. http:// www.dh.gov.uk/prod_consum_dh/groups/ dh_digitalassets/@dh/@en/documents/digitalasset/ dh_4137607.pdf (accessed 22 Aug 2011).

7. Ned RM, Sijbrands EJ. Cascade screening for familial hypercholesterolemia (FH). PLoS Curr 2011;3 RRN1238.

8. Samani NJ, Tomaszewski M, Schunkert H. The personal genome-the future of personalised medicine? Lancet 2010;375:1497-8.

9. Dequeker E, Ramsden S, Grody WW, et al. Quality control in molecular genetic testing. Nat Rev Genet 2001;2:717-23.

10. Palomaki GE, Bradley LA, Richards CS, et al. Analytic validity of cystic fibrosis testing: a preliminary estimate. Genet Med 2003:5:15-20.
11. Hudson KL, Murphy JA, Kaufman DJ, et al. Oversight of US genetic testing laboratories. Nat Biotechnol 2006;24:1083-90.

12. Seneca S, Morris MA, Patton $\mathbf{S}$, et al. Experience and outcome of 3 years of a European EQA scheme for genetic testing of the spinocerebellar ataxias. Eur J Hum Genet 2008;16:913-20.

13. Hertzberg M, Neville S, McDonald D. External quality assurance of molecular analysis of haemochromatosis gene mutations. J Clin Pathol 2006;59:744-7.

14. Hofgartner WT, Tait JF. Frequency of problems during clinical molecular-genetic testing. Am J Clin Pathol 1999;112:14-21.

15. Bonini $\mathbf{P}$, Plebani $M$, Ceriotti $F$, et al. Errors in laboratory medicine. Clin Chem 2002;48:691-8.

16. Dzik WH, Murphy MF, Andreu G, et al. An international study of the performance of sample collection from patients. Vox Sanguis 2003;85:40-7.

17. Murphy MF, Stearn BE, Dzik WH. Current performance of patient sample collection in the UK Transfus Med 2004;14:113-21.

18. Grimm E, Friedberg RC, Wilkinson DS, et al. Blood bank safety practices: mislabeled samples and wrong blood in tube-a 0-Probes analysis of 122 clinical laboratories. Arch Pathol Lab Med 2010;134:1108-15.

19. Lumadue JA, Boyd JS, Ness PM. Adherence to strict specimen-labeling policy decreases the incidence of erroneous blood grouping of blood bank specimens. Transfusion 1997:37:1169-72.

20. Howanitz PJ, Walker K, Bachner P. Quantification of errors in laboratory reports. A quality improvement study of the College of American Pathologists' Q-Probes program. Arch Pathol Lab Med 1992;116:694-700.

21. Nakhleh RE, Zarbo RJ. Surgical pathology specimen identification and accessioning: a College of American Pathologists 0-Probes Study of 1,004,115 cases from 417 institutions. Arch Pathol Lab Med 1996; 120:227-33.

22. Figueroa PI, Ziman A, Wheeler C, et al. Nearly two decades using the check-type to prevent $A B O$ incompatible transfusions: one institution's experience. Am J Clin Pathol 2006:126:422-6.

23. Houtz T, Chiafari FA, Wenk RE. Controlling specimen misidentification in parentage analysis. Transfusion 2004:44:1258-9.

24. Astion ML, Shojania KG, Hamill TR, et al. Classifying laboratory incident reports to identify problems that jeopardize patient safety. Am J Clin Pathol 2003;120:18-26.

25. Carraro $\mathbf{P}$, Plebani M. Errors in a stat laboratory: types and frequencies 10 years later. Clin Chem 2007; 53:1338-42.

26. The Joint Commission. Accreditation Program Laboratory National Patient Safety Goals. 2011. http://www.jointcommission.org/assets/1/6/ 2011 NPSGs LAB.pdf (accessed 2 Aug 2011).

27. Dzik WH. New technology for transfusion safety. Br J Haematol 2007:136:181-90.

28. Hurley CK, Wade JA, Oudshoorn M, et al. A specia report: histocompatability testing guidelines for hematopoietic stem cell transplantation using volunteer donors. Hum Immunol 1999;60:347-60.

29. Ibarreta D, Elles R, Cassiman JJ, et al. Towards quality assurance and harmonization of genetic testing services in the European Union. Nat Biotechnol 2004;22:1230-5.

30. McGovern MM, Elles R, Beretta I, et al. Report of an international survey of molecular genetic testing laboratories. Community Genet 2007;10:123-31.

31. Rainen L, Arbique JC, Asthana D, et al. Collection, Transport, Preparation, and Storage of Specimens for Molecular Methods; Proposed Guideline. Wayne, Pennsylvania USA: Clinical and laboratory standards Institute (CLSI), 2005. CLSI Document No. MM13-P.

32. Richards S, Grody WW, Dequeker E, et al. Molecular Diagnostic Methods for Genetic Diseases; Approved Guideline. 2nd edn. Wayne, Pennsylvania USA:
Clinical and laboratory standards Institute (CLSI), 2006. CLSI document MM1-A2.

33. American College of Medical Genetics. Standards and Guidelines for Clinical Genetics Laboratories. 2006 http://www.acmg.net/Pages/ACMG_Activities/stds2002/g.htm (accessed 2 Aug 2011).

34. Organization for Economic Cooperation and Development (OECD). OECD Guidelines for Quality Assurance in Molecular Genetic Testing. Paris France, 2007. http://www.oecd.org/dataoecd/43/6/ 38839788.pdf (accessed 2 Aug 2011).

35. Javaher $\mathbf{P}$, Kaariainen $\mathrm{H}$, Kristoffersson $\mathrm{U}$, et al. EuroGentest: DNA-based testing for heritable disorders in Europe. Community Genet 2008;11:75-120.

36. Chen B, Gagnon M, Shahangian S, et al. Good laboratory practices for molecular genetic testing for heritable diseases and conditions. MMWR Recomm Rep 2009;58:1-37.

37. Dequeker E, Stuhrmann M, Morris MA, et al. Best practice guidelines for molecular genetic diagnosis of cystic fibrosis and CFTR-related disorders-updated European recommendations. Eur J Hum Genet 2009;17:51-65

38. Mattocks CJ, Morris MA, Matthijs G, et al. A standardized framework for the validation and verification of clinical molecular genetic tests. Eur $\mathrm{J}$ Hum Genet 2010;18:1276-88.

39. Harper JC, Sengupta S, Vesela K, et al. Accreditation of the PGD laboratory. Hum Reprod 2010;25:1051-65

40. Plebani M. Exploring the iceberg of errors in laboratory medicine. Clin Chim Acta 2009;404:16-23.

41. Royal College of Pathologists of Australasia. Suthers G, Yu S in Report of the Australian Genetic Testing Survey. 2006. http://www.rcpa.edu.au/static/ File/Asset library/public documents/Media Releases/ AustralianGeneSurvey2006.pdf (accessed 2 Aug 2011).

42. National Pathology Accreditation Advisory Council, Australian Government Department of health and Aging. Laboratory Accreditation Standards and Guidelines for Nucleic Acid Detection and Analysis. 2006. http://www.health.gov.au/ internet/main/publishing.nsf/Content/ 11E45788D881EE37CA25732B0048D60C/\$File/ NucAcidDetect.pdf (accessed 2 Aug 2011).

43. Vincent FHR. Report: Inquiry into the Circumstances That Led to the Conviction of Mr Farah Abdulkadir JAMA. Melbourne, Australia: Victorian Government Printer, 2010. PP No 301:Session 2006-10.

44. Murphy MF, Kay JD. Patient identification: problems and potential solutions. Vox Sang 2004;87/Suppl 2):197-202

45. Stainsby D, Russell J, Cohen $\mathrm{H}$, et al. Reducing adverse events in blood transfusion. $\mathrm{Br} \mathrm{J}$ Haematology 2005;131:8-12

46. Maddalena A, Bale S, Das S, et al; the ACMG Laboratory Quality Assurance Committee. Technical standards and guidelines: molecular genetic testing for ultra-rare disorders. Genet Med 2005; 7:571-84.

47. Belgian Society of Human Genetics. Guidelines for Predictive Genetic Testing for Late Onset Disorders. 2003. http://www.beshg.be/workgroups/ guidelinespredictivetesting.htm (accessed 2 Aug 2011)

48. Royal College of Pathologists of Australasia Position Statement. Sample requirements for medical genetic testing: do genetic tests demand a different standard? 2007. http://www.rcpa.edu.au/ static/File/Asset library/public documents/Policy Manual/Position Statements/Sample requirements for medical genetic testing.pdf (accessed 2 Aug 2011).

49. Thomas DL, Thio CL, Martin MP, et al. Genetic variation in IL28B and spontaneous clearance of hepatitis C virus. Nature 2009;461:798-801. 